\title{
Evolution of prostate cancer diagnosis: retrospective analysis of magnetic resonance imaging/ultrasound fusion guided biopsies protocol in routine practice and patients management
}

\author{
Mohamed Ali Essid ${ }^{1}$, Marouene Chakroun ${ }^{1}$, François Xavier Nouhaud ${ }^{1}$, Michael Lair ${ }^{2}$, Françoise Gobet ${ }^{3}$, \\ Christian Pfister ${ }^{1,4}$ \\ ${ }^{1}$ Department of Urology, ${ }^{2}$ Department of Radiology, ${ }^{3}$ Department of Pathology, Rouen University Hospital, Rouen, France; ${ }^{4}$ Clinical Investigation \\ Center, Inserm 6204, Onco-Urology, Rouen, France \\ Contributions: (I) Conception and design: MA Essid, M Chakroun, C Pfister; (II) Administrative support: C Pfister; (III) Provision of study materials \\ or patients: MA Essid, M Chakroun, FX Nouhaud, C Pfister; (IV) Collection and assembly of data: MA Essid, M Chakroun, FX Nouhaud, C Pfister; \\ (V) Data analysis and interpretation: M Lair, F Gobet; (VI) Manuscript writing: All authors; (VII) Final approval of manuscript: All authors. \\ Correspondence to: Christian Pfister, Department of Urology, Charles Nicolle Rouen University Hospital, 1 rue de Germont, 76031Rouen Cedex, \\ France. Email: Christian.Pfister@chu-rouen.fr.
}

\begin{abstract}
Background: Magnetic resonance imaging (MRI) is today strongly recommended in prostate cancer (PCa) diagnosis. Therefore, MRI/ultrasound (MRI/US) fusion-guided biopsy is becoming the new standard patients management.

Methods: We report our experience during the last 4 years using this technique, with a protocol of 6 random cores (instead of the most used 12 cores protocol) associated to the target cores (2 to 3 per lesion). Our study involved 236 patients including real life routine practice: biopsy naïve patients $(\mathrm{n}=107)$, patients with previous negative standard prostate biopsies $(n=67)$ and patients in PCa active surveillance $(n=62)$. Finally, 76 patients have a robotic radical prostatectomy.

Results: Mean age of the population was 66 years. Median PSA was $8.5 \mathrm{ng} / \mathrm{mL}$. Overall and significant cancer detection were respectively $66.6 \%$ and $38.5 \%$, with a large difference considering biopsy history: $63.5 \%$ in biopsy naïve patient, $53.7 \%$ in patient with previous negative biopsies and $82.3 \%$ in patients under active surveillance. Targeted biopsies missed 28 cancers among 8 were significant and standard biopsies missed 33 cancers among 14 were significant. Moreover, concordance between biopsy samples and radical prostatectomy specimens was evaluated at $80 \%$.

Conclusions: Comparing to literature data, similar results were observed in our retrospective study, even with reduced random cores, suggesting a real change in patients management in particular in active surveillance group with a reclassification rate of 56.4\% using the Epstein criteria.
\end{abstract}

Keywords: Prostate cancer (PCa); magnetic resonance imaging/ultrasound fusion-guided biopsy (MRI/US fusion-guided biopsy)

Submitted Jul 22, 2019. Accepted for publication Nov 29, 2019.

doi: $10.21037 /$ tau.2020.02.02

View this article at: http://dx.doi.org/10.21037/tau.2020.02.02

\section{Introduction}

Despite transrectal ultrasound guided systematic prostate biopsy is the standard-of-care in diagnosis of prostate cancer (PCa), it has poor diagnostic accuracy for PCa detection (1). It may fail to detect the presence of cancer due its low sensitivity and specificity (2), so that the false-negative rate may reach $50 \%$ (3). Furthermore, $30 \%$ of aggressive tumors are usually misdiagnosed (4).

The ability to detect PCa on magnetic resonance imaging (MRI), using different prognostic scores and systems (PI- 
RADS) has led to the development of three MRI-guided prostate biopsy methods: cognitive fusion biopsy, MRI/ ultrasound (MRI/US) fusion-guided biopsy and biopsy performed in gantry in an MRI suite (5). As the first one lacks of precision and depends on the operator's expertise, and the third one is still expensive at the moment, MRI/ US fusion biopsy is being embraced as the new standard technique (6). Nevertheless, there is no consensus for the moment about the optimal protocol of biopsies to perform in PCa initial diagnosis.

The objective of this retrospective study was to report the results of our experience in real life routine practice using the MRI/ultrasound imaging biopsy technique with 6 random cores associated to targeted cores ( 2 to 3 per lesion), with comparison to the main publications of the literature.

\section{Methods}

In this retrospective study, we reported our experience of MRI/US fusion biopsy in an academic reference urological center, during a 4 years period from September 2015 to May 2019.

There were no exclusion criteria based on age, PSA, prostate volume, previous history of prostate biopsy. All patients have at least one suspicious lesion on multiparametric MRI (mp-MRI).

Subsequently to fusion biopsy, histological results of radical prostatectomy specimens were also analyzed. An ethical waiver was applicable as the study was a retrospective service evaluation without any change in patient management.

\section{Multiparametric MRI}

A mp-MRI was performed for all patients, including T2weighted imaging (T2w), diffusion-weighted imaging (DWI) with apparent diffusion coefficient (ADC) reconstructions, and dynamic contrast enhanced (DCE) imaging. All MRIs data were reviewed by a single referring uro-radiologist (M Lair), and suspicious lesions were scored according to the PI-RADS classification (version 2) (7).

\section{Biopsies procedure}

Fusion biopsies were performed with the UroStation ${ }^{\mathrm{TM}}$ (Koelis, Grenoble, France). Suspect lesions (PI-RADS $\geq 3$ ) were previously contoured and recorded by our radiologist
(M Lair). All procedures were performed under local anesthesia, by two urologists experienced in PCa. During the procedure, we always realized 2 to 3 targeted biopsies on each suspect lesion associated to a sextant scheme of six random biopsies (one core, bilaterally from each base, mid and apex).

All biopsies and prostatectomy specimens after radical prostatectomy were analyzed by an experienced uropathologist (F Gobet). To date, there is always a debate about the definition of significant PCa. Anyway, we considered EAU guidelines definition of a PCa with a Gleason 7 or higher score (1).

\section{Statistical analysis}

Descriptive statistics were performed to summarize clinical, MRI data, fusion biopsy samples and prostatectomy specimens' characteristics. Spearman correlation coefficient was performed to assess the Gleason's score concordance between fusion biopsy samples and prostatectomy specimens.

\section{Results}

A total of 244 patients with MRI/US fusion prostate biopsy were reported during the study period. During the same period, 444 standard prostate biopsies were realized in our department. The interest of Figure 1 was to underline the evolution of the two biopsies techniques during the study period. Eight patients were excluded from the analysis because they had only targeted biopsies without random ones associated.

In real life routine practice, we stratified the included 236 patients into 3 groups: 107 biopsy naïve patients, 67 patients with previous negative standard prostate biopsies and 62 patients under PCa active surveillance.

The general characteristics of the patients and mp-MRI features are shown in Table 1. Mean age of the patients was 66 years (48 to 84). Median PSA was $8.5 \mathrm{ng} / \mathrm{mL}$. Only 26 patients had an abnormal digital rectal exam. According to MRI data, median prostate volume was $62 \mathrm{~cm}^{3}$. A single lesion was noticed in 192 (81.4\%) cases and 44 (18.6\%) patients had two or more lesions. A total of 294 suspects mp-MRI lesions were described: 62 PI-RADS 3 lesions (21\%), 154 PI-RADS 4 lesions (52.50\%), and 78 PI-RADS 5 lesions (26.5\%). Mean size of lesions was $12.5 \mathrm{~mm}$. A mean of 4.9 (2 to 12) targeted biopsies were carried out per patient. Biopsy and radical prostatectomy outcomes 


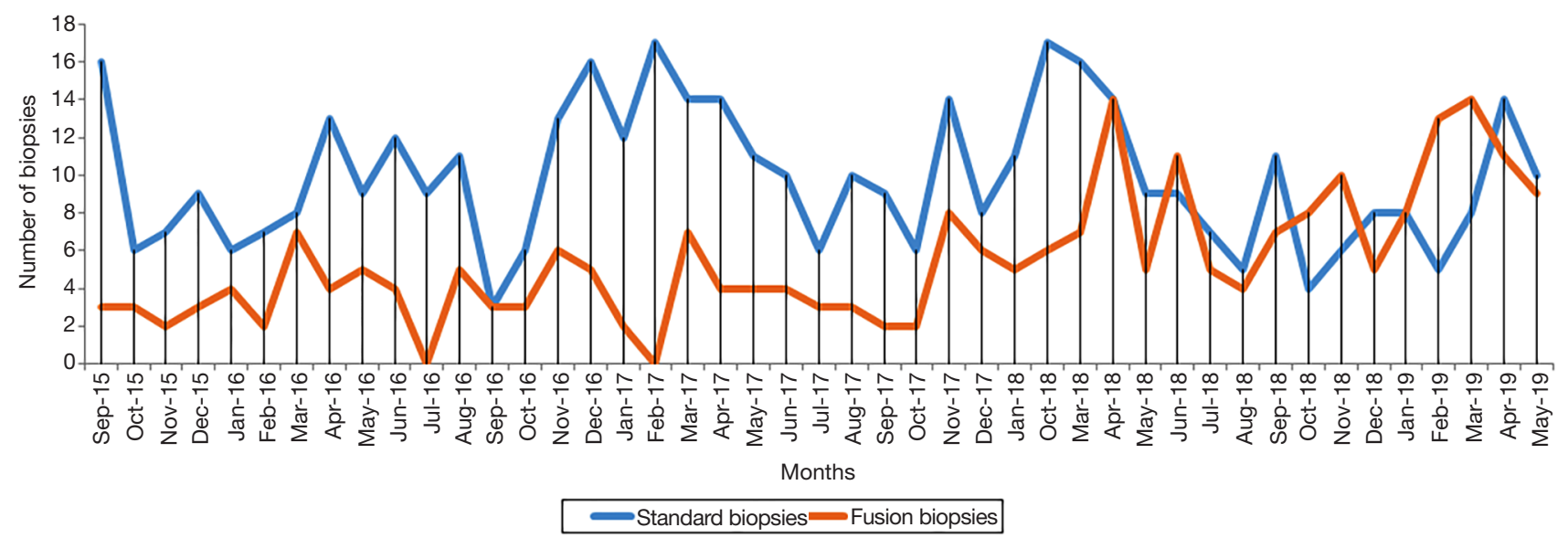

Figure 1 Monthly evolution of the number of standard and fusion biopsies during the study.

Table 1 Patients characteristics and mpMRI features

\begin{tabular}{lc}
\hline Parameters & Values \\
\hline Men included (all $\mathrm{n}=244)$ & 236 \\
Mean age (years) [range] & $66[48-84]$ \\
Mean PSA (ng/mL) [range] & $8.5[0.5-80]$ \\
Mean prostate volume (cc) [range] & $62[15-270]$ \\
Family history of prostate cancer, $\mathrm{n}(\%)$ & $26(11.0)$ \\
Abnormal digital rectal examination, $\mathrm{n}(\%)$ & $26(11.0)$ \\
Biopsies history, $\mathrm{n}$ (\%) & \\
Biopsy naïve & $107(45.3)$ \\
Previous negative biopsies & $67(28.4)$ \\
Patients in active surveillance & $62(26.3)$ \\
PI-RADS targets (maximum score in case of multiple targets), $\mathrm{n}(\%)$ \\
PI-RADS 3 & $38(16.1)$ \\
PI-RADS 4 & $125(53.0)$ \\
PI-RADS 5 & $73(30.9)$ \\
Number of biopsy cores & $12.5[3-30]$ \\
Standard & $1,912-12]$ \\
Targeted & \\
Mean of targeted biopsies per patient [range] & 4.90 \\
mpMRI targets per patient & \\
Mingle & \\
\hline Multiple & \\
\hline
\end{tabular}

are reported in Table 2. The overall cancer detection rate (CDR) was $66.6 \%$, and down to $38.5 \%$ if only considering clinically significant cancer.

As shown in the Figure 2, the PI-RADS score was associated with the presence of PCa as $84.7 \%, 64 \%$ and $34.2 \%$ of respectively PI-RADS 5, PI-RADS 4 and PIRADS 3 lesions biopsies were positive, as well as the presence of significant $\mathrm{PCa}$ (respectively $64.3 \%, 32 \%$ and $10.5 \%)$. PCa was detected only in random and targeted biopsies respectively in $28(18 \%)$ and $33(21.3 \%)$ cases. It was detected in both biopsies in 94 (60.6\%) cases. Targeted biopsies missed 28 cancers among 8 were significant and standard biopsies missed 33 cancers 14 were significant (Figure 3).

CDR was widely different depending on the patient history. Therefore, CDR was $63.5 \%$ in biopsy naïve patient, $53.7 \%$ in patient with previous negative biopsies and $82.3 \%$ in patients in active surveillance. When considering significant cancer definition, biopsy naïve and active surveillance groups toward to have similar CDR $(42.9 \%$ and $43.5 \%$ respectively), as patient with previous negative biopsies have lower but none negligible CDR (25.3\%).

Among the 62 patients in active surveillance and in consideration with Epstein Criteria, 35 (56.4\%) were eligible to an active treatment after undergoing fusion biopsy. Robot-assisted radical prostatectomy was performed in 76 men. Considering the whole Gleason score, concordance between biopsy samples and radical prostatectomy specimens was $80 \%$ (Figure 4). The Spearman correlation coefficient showed a very strong and 
Table 2 Biopsy and prostatectomy histological results

\begin{tabular}{|c|c|}
\hline Parameters & Values \\
\hline On targeted biopsy only & $33(14.0)$ \\
\hline On random biospy only & $28(11.9)$ \\
\hline On both biopsy & $94(39.8)$ \\
\hline On targeted biopsy only & $14(5.9)$ \\
\hline On random biospy only & $8(3.4)$ \\
\hline On both biopsy & $68(28.8)$ \\
\hline Overall cancer detection rate, $\mathrm{n}(\%)$ & $155(65.7)$ \\
\hline Patients in active surveillance & $51 / 62(82.3)$ \\
\hline Significant cancer detection rate, $\mathrm{n}(\%)$ & $90(38.1)$ \\
\hline Biopsy naïve & $46 / 107(43.0)$ \\
\hline Previous negative biopsies & $17 / 67(25.4)$ \\
\hline Patients in active surveillance & $27 / 62(43.5)$ \\
\hline Robot-assisted radical prostatectomy & 76 \\
\hline Gleason score concordance between fusion biopsy samples between radical prostatectomy specimen & $60 / 76(78.9)$ \\
\hline Gleason 6 reclassement from biopsy to radical prostatectomy specimen & $11 / 25(44.0)$ \\
\hline
\end{tabular}

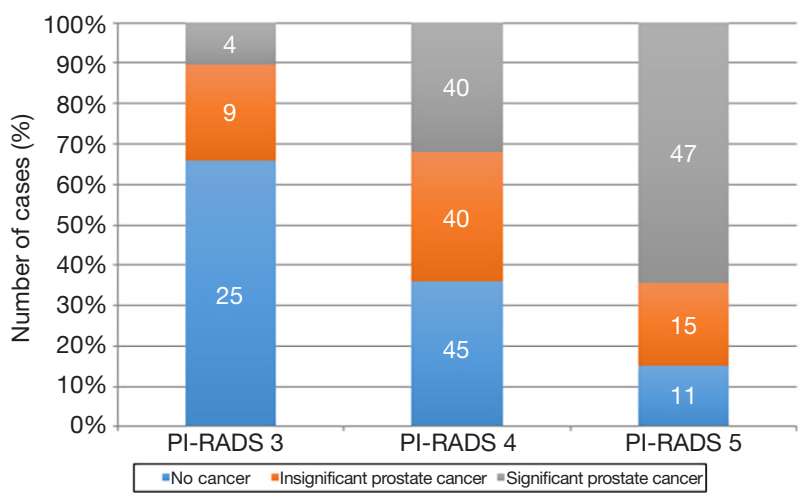

Figure 2 Overall $\mathrm{PCa}$ and significant $\mathrm{PCa}$ detection rate according to PI-RADS score.

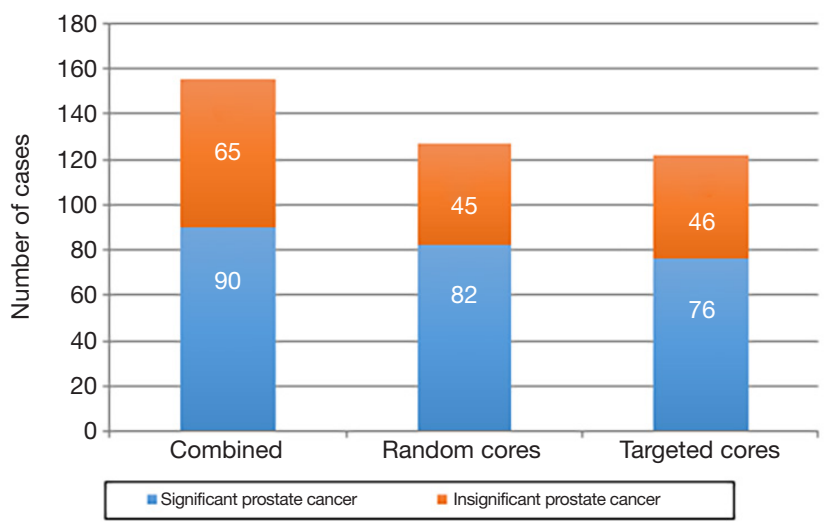

Figure 3 PCa detection according to the combined and separated biopsy. 


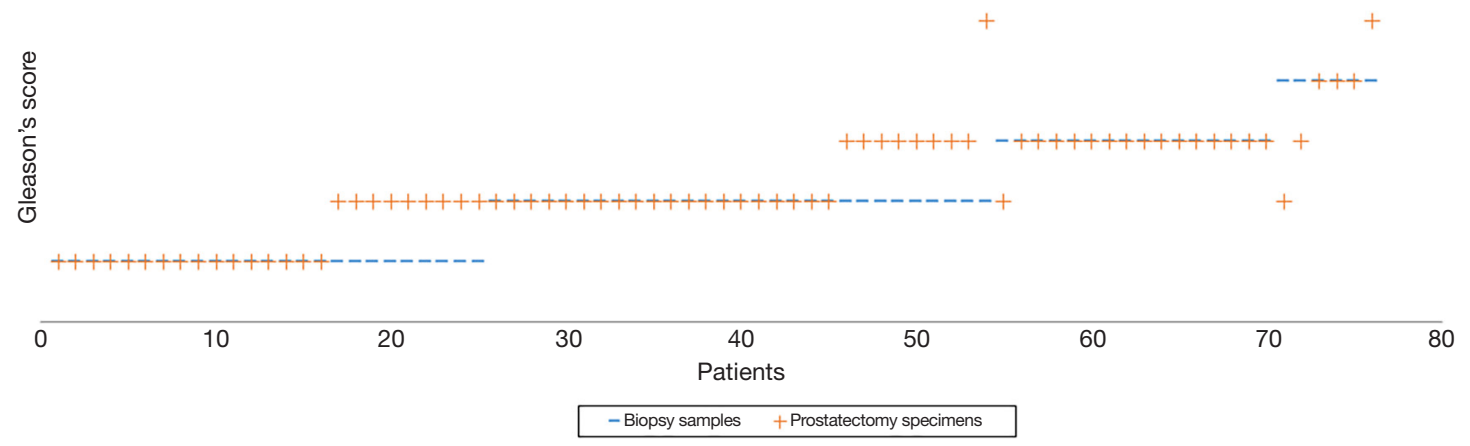

Figure 4 Concordance of Gleason score between biopsy samples and prostatectomy specimens.

significant positive correlation between the two histological scores $(\mathrm{P}=0.81, \mathrm{P}<0.001)$. As regarding patients with Gleason 6 on biopsies samples, $44 \%$ were upgraded to Gleason 7 or higher on radical prostatectomy specimen.

\section{Discussion}

In our retrospective study, overall CDR was $63.5 \%$, which were slightly higher than other fusion biopsies studies data $(8,9)$. This is probably related to the heterogeneity of the study population with an important group of PCa patients already diagnosed and under active surveillance. In fact, across studies, PCa detection rate was variable and was influenced by patient selection study designs and reporting which made difficult a head-to head comparison.

In the early 2000's, saturation biopsies were proposed to patients with previous negative biopsies but raising PSA and clinical suspicion for PCa. More recently, the advent of multiparametric MRI permits to identify suspicious prostate lesions with a high accuracy. Different MRI classification scores were validated to objectively grade those suspicious lesions. PI-RADS (version 2) remains today the most used in clinical practice, with the advantage, in plus of its accuracy, to present an overall good inter-observer agreement among radiologists with different levels of experience (10). These radiological data suggest to proposed biopsies targeting directly suspicious lesions, rather than repeated systematic biopsy. As reported by Sonn et al. fusion biopsy provides improved detection of $\mathrm{PCa}$ in men with prior negative biopsies with an overall CDR of 34\% (11). Anyway, the reported $\mathrm{CDR}$ for significant $\mathrm{PCa}$ in men with a prior negative biopsy can range between 20 and $40 \%$ and is lower compared to detection rate in biopsy-naive patients $(12,13)$.

Different series have evaluated the interest of performing targeted biopsies in primary intention for naïve patients. Mozer et al., in a series of 152 patients with a control arm of conventional echo-guided biopsies, concluded that biopsies targeted with Urostation (Koelis ${ }^{\circledR}$ ) have detected a lower number of cancers at all grades (54\% vs. 57\%), but more lesions clinically significant (43.4\% vs. $36.8 \%$ ) (14). Similar results have been reported by Pokorny et al. with a higher high-risk carcinoma rate in the targeted biopsy group (65.5\% vs. 52.1\%) (15). Using another software (Virtual Navigator), Delongchamps et al. observed a significantly higher CDR $(82.1 \%$ vs. $45.8 \%)$, with a higher Gleason score 7 rate than in the targeted biopsy group (16). A recent international study, PROMIS, has underlined that performing an initial selection of patients by MRI before any biopsy could potentially avoid the practice of biopsies in $27 \%$ of patients, and would guide the subsequent biopsy for patient with suspicious lesions (17). Anyway, these literature data are similar to our retrospective study showing a CDR at biopsy naïve men of $63.5 \%$, clinically significant in $42.9 \%$.

In contrast, in active surveillance the reclassification rate was much lower, ranging from $2.5 \%$ for Soloway et al. (18), up to $22 \%$ for Berglund et al. (19). Therefore, those findings would indicate mp-MRI and targeted biopsy at initial evaluation during an active surveillance protocol to offer more relevant detection of significant cancers (20). On a series of 113 patients, $\mathrm{Hu}$ et al. noted a reclassification rate of 36\% according to the Epstein criteria (Gleason score less than 7 , invasion of less than 3 cores and/or tumor extent less than $50 \%$ per carrot) (21). Using the same criteria, reclassification rate in our study would be $56.4 \%$. Nahar et al. showed that targeted biopsies made $33 \%$ of patient ineligible to active surveillance considering Epstein criteria (22). Half of those patients were ineligible because of Gleason upgrading. 
Should we systematically combine standard biopsies with targeted biopsies in routine practice? From a morbidity point of view, no series has shown a significant decrease of complications by reducing the number of samples. Nevertheless, the number of samples seems to be correlate with less patient pain. Siddiqui et al. observed the same detection rate for overall prostate carcinoma between standard biopsies and targeted biopsies, but the latter allowed diagnosing $30 \%$ of high-risk tumors and $17 \%$ fewer tumors low risk (23). Our results are also in concordance with the literature data. Rouvière $e t$ al. in the "MRI-First" study concluded that significant CDR was improved by combining both techniques and both techniques showed substantial added value (24). In our experience, only $8 \%$ of significant cancers were diagnosed on randomized samples. Many studies have reported that the percentage of clinically significant cancer missed by fusion biopsy, but detected by standard biopsy could reach to $10 \%(23,25)$. Such data clearly confirm the necessity of combining target and random biopsies.

Recently, Aminsharifi et al. have evaluated the six random cores protocol and showed an increased efficiency of detecting clinically-significant PCa even with fewer random cores (26). Siddiqui et al. reported that performing exclusively targeted biopsies allowed diagnosis of the same rate of $\mathrm{PCa}$ as combined biopsies, with higher significant cancer rates, and fewer samples (57\%) (27). As well, the randomized PRECISION study compared two groups of biopsy naive men: the MRI-targeted biopsy group (without random biopsy cores) versus standard biopsy group. They concluded that even if targeted biopsy alone misses a low rate of significant cancer, this procedure remains superior to standard biopsy, with the diagnosis of more significant cancer and fewer insignificant cancer (28).

Concerning surgery outcomes, Velez et al. reported their histological results on patients who had radical prostatectomy after fusion biopsy (29). Gleason scores were unchanged in $71 \%$ of cases with a Spearman correlation coefficient of 0.75 , which is comparable to our study results. Bloom et al. reported a rate of $44 \%$ of upgrade among their 466 Gleason 6 patients (25). In the other hand, some studies concluded that MRI/US biopsy besides their higher significant PCa detection rate comparing to standard biopsy, have also less upgrading of cancers at surgery $(30,31)$.

\section{Conclusions}

In conclusion, targeted biopsies led to increase clinically significant CDR, which has been consistently reported across cohort studies despite wide variations in MRI protocols, patient selection and operator technique. In our series, CDR was $63.5 \%$ in biopsy naïve patient, $53.7 \%$ in patient with previous negative biopsies and $82.3 \%$ in patients in active surveillance. Moreover, targeted prostate biopsy is tending to become the gold standard in the management of patients with PCa suspicion. Our study demonstrated in comparison with literature data that a reduced number of random cores could be associated with similar results, suggesting a real change in patient's management in particular in active surveillance group. Using the Epstein criteria, reclassification rate in our study would be $56.4 \%$.

\section{Acknowledgments}

Funding: None.

\section{Footnote}

Conflicts of Interest: All authors have completed the ICMJE uniform disclosure form (available at http://dx.doi. org/10.21037/tau.2020.02.02). The authors have no conflicts of interest to declare.

Ethical Statement: The authors are accountable for all aspects of the work in ensuring that questions related to the accuracy or integrity of any part of the work are appropriately investigated and resolved. An ethical waiver was applicable as the study was a retrospective service evaluation without any change in patient management.

Open Access Statement: This is an Open Access article distributed in accordance with the Creative Commons Attribution-NonCommercial-NoDerivs 4.0 International License (CC BY-NC-ND 4.0), which permits the noncommercial replication and distribution of the article with the strict proviso that no changes or edits are made and the original work is properly cited (including links to both the formal publication through the relevant DOI and the license). See: https://creativecommons.org/licenses/by-ncnd/4.0/.

\section{References}

1. Mottet N, Bellmunt J, Bolla M, et al. EAU-ESTROSIOG guidelines on prostate cancer Part 1: screening, 
diagnosis, and local treatment with curative intent. Eur Urol 2017;71:618-29.

2. Center MM, Jemal A, Lortet-Tieulent J, et al. International variation in prostate cancer incidence and mortality rates. Eur Urol 2012;61:1079-92.

3. Roobol MJ, Kranse R, Bangma CH, et al. Screening for prostate cancer: results of the Rotterdam section of the European Randomized Study of Screening for Prostate Cancer. Eur Urol 2013;64:530-9.

4. Loeb S, Bjurlin MA, Nicholson J, et al. Overdiagnosis and overtreatment of prostate cancer. Eur Urol 2014;65:1046-55.

5. Kuru TH, Herden J, Zugor V, et al. How to Perform Image-guided Prostate Biopsy: In-bore and Fusion Approaches. Eur Urol Focus 2016;2:151-3.

6. Boesen L. Magnetic resonance imaging-transrectal ultrasound image fusion guidance of prostate biopsies: current status, challenges and future perspectives. Scand J Urol 2019;53:89-96.

7. Barentsz JO, Weinreb JC, Verma S, et al. Synopsis of the PI-RADS v2 guidelines for multipa $\neg$ rametric prostate magnetic resonance imaging and recommenda $\neg$ tions for use. Eur Urol 2016;69:41-9.

8. Filson CP, Natarajan S, Margolis DJ, et al. Prostate cancer detection with MR-ultrasound fusion biopsy: the role of systematic and targeted biopsies. Cancer 2016;122:884.

9. Oderda M, Marra G, Albisinni S, et al. Accuracy of elastic fusion biopsy in daily practice: Results of a multicenter study of 2115 patients. Int J Urol 2018;25:990-97.

10. Mussi TC, Yamauchi FI, Tridente CF, et al. Interobserver Agreement and Positivity of PI-RADS Version 2 Among Radiologists with Different Levels of Experience. Acad Radiol 2019;26:1017-22.

11. Sonn GA, Chang E, Natarajan S, et al. Value of targeted prostate biopsy using magnetic resonance-ultrasound fusion in men with prior negative biopsy and elevated prostate-specific antigen. Eur Urol 2014;65:809-15.

12. Schoots IG, Roobol MJ, Nieboer D, et al. Magnetic resonance imaging-targeted biopsy may enhance the diagnostic accuracy of significant prostate cancer detection compared to standard transrectal ultrasound-guided biopsy: a systematic review and meta-analysis. Eur Urol 2015;68:438-50.

13. Fütterer JJ, Verma S, Hambrock T, et al. High-risk prostate cancer: value of multi-modality $3 \mathrm{~T}$ MRI-guided biopsies after previous negative biopsies. Abdom Imaging 2012;37:892-6.

14. Mozer P, Rouprêt M, Le Cossec C, et al. First round of targeted biopsies with magnetic resonance imaging/ ultrasound-fusion images compared to conventional ultrasound-guided trans-rectal biopsies for the diagnosis of localised prostate cancer. BJU Int 2015;115:50-7.

15. Pokorny MR, de Rooij M, Duncan E, et al. Prospective study of diagnostic accuracy comparing prostate cancer detection by transrectal ultrasound-guided biopsy versus magnetic resonance (MR) imaging with subsequent MRguided biopsy in men without previous prostate biopsies. Eur Urol 2014;66:22-9.

16. Delongchamps NB, Peyromaure M, Schull A, et al. Prebiopsy magnetic resonance imaging and prostate cancer detection: comparison of random and targeted biopsies. J Urol 2013; 189: 493-9.

17. Ahmed HU, El-Shater Bosaily A, Brown LC, et al. Diagnostic accuracy of multi-parametric MRI and TRUS biopsy in prostate cancer: a paired validating confirmatory study. Lancet 2017;389:815-22.

18. Soloway MS, Soloway CT, Eldefrawy A, et al. Careful selection and close monitoring of low-risk prostate cancer patients on active surveillance minimizes the need for treatment. Eur Urol 2010;58:831-35.

19. Berglund RK, Masterson TA, Vora KC, et al. Pathological upgrading and up staging with immediate repeat biopsy in patients eligible for active surveillance. J Urol 2008;180:1964-67.

20. Denize J, Nouhaud FX, Cornu JN, et al. Re: Timing of Adverse Prostate Cancer Reclassification on First Surveillance Biopsy: Results from the Canary Prostate Cancer Active Surveillance Study. L. C. Macleod et al. J Urol 2017;198:709-11.

21. Hu Y, Ahmed HU, Taylor Z, et al. MR to ultrasound registration for image-guided prostate interventions. Med Image Anal 2012;16:687-703.

22. Nahar B, Katims A, Barboza MP, et al. Reclassification Rates of Patients Eligible for Active Surveillance After the Addition of Magnetic Resonance Imaging-Ultrasound Fusion Biopsy: An Analysis of 7 Widely Used Eligibility Criteria. Urology 2017;110:134-9.

23. Siddiqui MM, Rais-Bahrami S, Truong H, et al. Magnetic resonance imaging/ultrasound-fusion biopsy significantly upgrades prostate cancer versus systematic 12-core transrectal ultrasound biopsy. Eur Urol 2013;64:713-9.

24. Rouvière $\mathrm{O}$, Puech $\mathrm{P}$, Renard-Penna $\mathrm{R}$, et al. Use of prostate systematic and targeted biopsy on the basis of multiparametric MRI in biopsy-naive patients (MRIFIRST): a prospective, multicentre, paired diagnostic study. Lancet Oncol 2019;20:100-09. 
25. Bloom JB, Hale GR, Gold SA, et al. Predicting Gleason Group Progression for Men on Prostate Cancer Active Surveillance: Role of a Negative Confirmatory Magnetic Resonance Imaging-Ultrasound Fusion Biopsy. J Urol 2019;201:84-90.

26. Aminsharifi A, Gupta RT, Tsivian E, et al. Reduced Core Targeted (RCT) biopsy: Combining multiparametric magnetic resonance imaging - transrectal ultrasound fusion targeted biopsy with laterally-directed sextant biopsies An alternative template for prostate fusion biopsy. Eur J Radiol 2019;110:7-13.

27. Siddiqui MM, George AK, Rubin R, et al. Efficiency of Prostate Cancer Diagnosis by MR/Ultrasound FusionGuided Biopsy vs. Standard Extended-Sextant Biopsy for MR-Visible Lesions. J Natl Cancer Inst 2016. doi: 10.1093/jnci/djw039.

28. Kasivisvanathan V, Rannikko AS, Borghi M, et al. MRI-

Cite this article as: Essid MA, Chakroun M, Nouhaud FX, Lair M, Gobet F, Pfister C. Evolution of prostate cancer diagnosis: retrospective analysis of magnetic resonance imaging/ ultrasound fusion guided biopsies protocol in routine practice and patients management. Transl Androl Urol 2020;9(2):629-636. doi: $10.21037 /$ tau.2020.02.02
Targeted or Standard Biopsy for Prostate-Cancer Diagnosis. N Engl J Med 2018;378:1767-77.

29. Velez E, Fedorov A, Tuncali K, et al. Pathologic correlation of transperineal in-bore 3-Tesla magnetic resonance imaging-guided prostate biopsy samples with radical prostatectomy specimen. Abdom Radiol (NY) 2017;42:2154-9.

30. Arsov C, Becker N, Rabenalt R, et al. The use of targeted MR-guided prostate biopsy reduces the risk of Gleason upgrading on radical prostatectomy. J Cancer Res Clin Oncol 2015;141:2061-68.

31. Salami SS, Ben-Levi E, Yaskiv O, et al. In patients with a previous negative prostate biopsy and a suspicious lesion on magnetic resonance imaging, is a 12-core biopsy still necessary in addition to a targeted biopsy? BJU Int 2015;115:562-70. 